\title{
The Nucleocapsid Protein of SARS-CoV-2 Abolished Pluripotency in Human Induced Pluripotent Stem Cells
}

\section{Zebin Lin ${ }^{1,2 \#, ~ Z h i m i n g ~ W u ~}{ }^{3 \#}$, Jinlian Mai ${ }^{2}$, Lishi Zhou ${ }^{2}$, Yu Qian ${ }^{1}$, Tian Cai ${ }^{4}$, Zhenhua Chen ${ }^{4}$,} Ping Wang ${ }^{5 *}$ and Bin Lin ${ }^{2 *}$

$3 \quad{ }^{1}$ School of Pharmaceutical Sciences, Sun Yat-Sen University, Guangzhou, China

$4 \quad{ }^{2}$ Guangdong Beating Origin Regenerative Medicine Co. Ltd., Foshan, China

$5 \quad{ }^{3}$ Department of Urology, Sun Yat-sen University Cancer Center, State Key Laboratory of Oncology

6 in South China, Collaborative Innovation Center for Cancer Medicine, Guangzhou, China

$7 \quad{ }^{4}$ Nanhai District People's Hospital of Foshan, Foshan, China

$8{ }^{5}$ School of Medical Imaging, Tianjin Medical University, Tianjin, China

$9 \quad$ \#These authors contributed equally to the work.

10 * Correspondence:

11 Ping Wang

12 pingwang@tmu.edu.cn;

13 Bin Lin

14 linbin@beatingorigin.com.

Keywords: SARS-CoV-2, nucleocapsid protein, human induced pluripotent stem cell,

17 pluripotency, fibroblast. 


\section{Abstract}

The COVID-19 pandemic caused by severe acute respiratory syndrome coronavirus 2 (SARS-CoV2 ) is raging across the world, leading to a global mortality rate of $3.4 \%$ (estimated by World Health Organization in March 2020). As a potential vaccine and therapeutic target, the nucleocapsid protein of SARS-CoV-2 (nCoVN) functions in packaging the viral genome and viral self-assembly. To investigate the biological effects of $\mathrm{nCoVN}$ to human stem cells, genetically engineered human induced pluripotent stem cells (iPSC) expressing nCoVN (iPSC-nCoVN) were generated by lentiviral expression systems, in which the expression of $\mathrm{nCoVN}$ could be induced by the doxycycline. The proliferation rate of iPSC-nCoVN was decreased. Unexpectedly, the morphology of iPSC started to change after nCoVN expression for 7 days. The pluripotency marker TRA-1-81 were not detectable in iPSC-nCoVN after a four-day induction. Meanwhile, iPSC-nCoVN lost the ability for differentiation into cardiomyocytes with a routine differentiation protocol. The RNA-seq data of iPSC-nCoVN (induction for 30 days) and immunofluorescence assays illustrated that iPSCnCoVN were turning to fibroblast-like cells. Our data suggested that nCoVN disrupted the pluripotent properties of iPSC and turned them into other types of cells, which provided a new insight to the pathogenic mechanism of SARS-CoV-2.

\section{Introduction}

Right now, the COVID-19 pandemic is sweeping the world, causing a huge crisis in public health and economics globally. According to the continuously updated data from World Health Organization, to date, nearly three million infected cases were confirmed, while more than 200,000 individuals died because of COVID-19 (https://www.who.int/emergencies/diseases/novelcoronavirus-2019). Severe acute respiratory syndrome coronavirus 2 (SARS-CoV-2), which was proved to be the pathogen of COVID-19, has $79 \%$ identity in genomes with severe acute respiratory syndrome coronavirus (SARS-CoV) (Lu et al., 2020). Twelve coding regions were predicted in SARS-CoV-2, including spike protein, nucleocapsid protein, envelope protein, and membrane protein ( $\mathrm{Lu}$ et al., 2020; Wu et al., 2020; Xu et al., 2020). The Cryo-EM structure of spike protein had been determined (Wrapp et al., 2020), and more and more evidences showed that the spike protein binds human ACE2 to entry into host cells (Hoffmann et al., 2020; Wrapp et al., 2020), which indicated that SARS-CoV-2 might share similar pathogenic mechanisms with SARS-CoV. Because of the very limited knowledge of SARS-CoV-2, we sought to understand the biology of SARS-CoV2 based on the previous studies about SARS-CoV.

As one of the most studied proteins in SARS-CoV, the nucleocapsid protein binds to viral RNA to package the genome in a ribonucleoprotein particle (Chang et al., 2014). Unlike the spike protein with a certain mutation frequency, the sequence of nucleocapsid protein was more stable (Chinese, 2004), which meant it was an ideal target for diagnostic tools (Severance et al., 2008; Suresh et al., 2008; Das et al., 2010) and antiviral therapy (Cheung et al., 2008; Chang et al., 2016). The pathogenic effects in host cells caused by the nucleocapsid protein were also studied. It was reported that the nucleocapsid protein inhibited type I interferon production after virion infected the host cells (Hu et al., 2017), which was considered as a possible mechanism of immune escape. The nucleocapsid protein inhibited cell cytokinesis and proliferation (Zhou et al., 2008), and regulated several pathways, such as transforming growth factor-beta signaling (Zhao et al., 2008), AP-1 signal transduction pathway (He et al., 2003), and NF-KappaB pathway (Zhang et al., 2007b). Besides, the nucleocapsid protein was reported as an apoptosis inducer in COS-1 cells (Surjit et al., 2004; Zhang et al., 2007a) and HPF cells (Zhao et al., 2006). 
As the nucleocapsid protein of SARS-CoV-2 (nCoVN) has $88.1 \%$ identity with the nucleocapsid protein of SARS-CoV ( $\mathrm{Lu}$ et al., 2020), it is reasonable to speculate that they share a same pathogenic pathway in host cells. The original goal of this study is to determine the physiological malfunctions, such as cardiac fibrosis, in human cardiomyocytes expressing nCoVN by using human induced pluripotent stem cells (iPSC) and direct cardiac-differentiation methods. However, the morphology of iPSC altered obviously when $\mathrm{nCoVN}$ had been expressed for 7 days. This unexpected observation inspired us some new thoughts: (i) It was likely that the adult stem cells could be infected by SARS-CoV-2, in spite of lacking of the clinical data; (ii) iPSC were appropriate study materials for stem cell research because of avoiding many ethical issues; (iii) The preliminary data indicated that nCoVN seemed to be deleterious to iPSC, which meant it might also cause damages in other stem cells. Therefore, we turned to investigate whether nCoVN obstructed the pluripotency maintenance in iPSC. We believed that this study could help us to understand the deleterious effects of $\mathrm{nCoVN}$ to human adult stem cells and embryonic stem cells.

\section{Method}

\subsection{Cell culture and differentiation assay}

Human induced pluripotent stem cells (iPSC) DYR0100 (from The American Type Culture Collection, ATCC) were plated on Matrigel matrix (hESC-Qualified, LDEV-Free, Corning, 354277)coated plates, and then were cultured in DMEM/F-12 medium (Gibco, 11320033) supplemented with STEMUP $^{\circledR}$ ES/iPS cell culture medium supplement (Nissan Chemical Corporation). STEMUP medium was changed every two days. iPSC were passaged every three to four days or when the cell culture was 80-90\% confluent. During passages, iPSC were rinsed with 1× DPBS (Gibco, 14040133) for one time then were treated with $0.5 \mathrm{mM}$ EDTA (Invitrogen, 15575020) in 1× DPBS (Gibco, 14190144) for 10 minutes at room temperature. The split ratio was 1:3-1:6. The detailed differentiation protocol was described in the previous published reports (Lin et al., 2017; Shekhar et al., 2018). Briefly, iPSC were treated with the small molecule CHIR99021 (Tocris, 4423, final concentration $10 \mu \mathrm{M}$ ) in the RPMI-BSA medium [RPMI 1640 Medium (HyClone, SH30027.01) supplemented with $213 \mu \mathrm{g} / \mathrm{ml}$ AA2P (1-ascorbic acid 2-phosphate magnesium) (Sigma, A8960) and $0.1 \%$ bovine serum albumin (BSA) (Sigma, A1470)] for 24 hours, then were incubated with RPMIBSA medium for 48 hours. On differentiation day 4, cells were treated with the small molecule IWP2 (Tocris, 3533, final concentration $5 \mu \mathrm{M}$ ) in RPMI-BSA medium. After 48 hours, the medium was changed to RPMI-BSA medium. Then, RPMI 1640 medium supplemented with 3\% KnockOut Serum Replacement (Gibco, 10828-028) was used to culture the cardiomyocytes in the following experiments. All the cells in this study (except iPSC-derived cardiomyocytes) were kept culturing in the STEMUP medium until they were applied to other assays.

\subsection{Generation of iPSC-nCoVN}

The cDNA of nCoVN with a N-terminal $6 \times$ His Tag coding sequence (GeneMedi) and puromycin resistance gene were sub-cloned into the plasmid pCW-Cas9-Blast (Addgene, 83481) to replace Cas9 and Blast cDNA, respectively. Lentivirus preparation using a third generation lentivirus packaging system were referred to the previous report (Jiang et al., 2015). We followed and modified the protocol from Zhang lab to detect MOI of the lentivirus and perform transduction (Shalem et al., 2014). After 24 hours of transduction, medium was changed to fresh STEMUP medium supplemented with doxycycline hyclate (Sigma, D9891) for induction. Two days later, puromycin (InvivoGen, ant-pr-1, final concentration $2 \mu \mathrm{g} / \mathrm{mL}$ ) was added into the STEMUP medium supplemented with doxycycline hyclate. After 2-3 days' selection, which resulted in a transduction efficiency of $\sim 30 \%$, single cell clones were manually picked and re-seeded in separated wells. For 
$117 \mathrm{nCoVN}$ expression induction, doxycycline hyclate (Sigma, D9891) was supplemented in the stem

118 cell culture medium at a final concentration of $2 \mu \mathrm{g} / \mathrm{mL}$, and the same amount of DMSO was added

119 to the stem cell culture medium for controls.

\section{$120 \quad 2.3$ Reverse transcription-PCR and Quantitative Real-time PCR}

121 Total RNA was extracted by using the UN1Q-10 Column Trizol Total RNA Isolation Kit (Sangon

122 Biotech, B511321-0100) prior to the treatment with DNase I (Sangon Biotech, B618252) for 30

123 minutes. mRNA was reverse transcribed by using iScript Reverse Transcription Supermix (Bio-Rad,

124 1708841). Quantitative Real-time PCR was performed by using a PikoReal Real-Time PCR

125 System (Thermo Fisher) with SsoAdvanced ${ }^{\mathrm{TM}}$ Universal SYBR ${ }^{\circledR}$ Green Supermix (Bio-Rad,

126 1725271). The primers for Reverse transcription-PCR and Quantitative Real-time PCR are as

127 followed (from $5^{\prime}$ to $3^{\prime}$ ):

128 ACE2-RT-F: GGTCTTCTGTCACCCGATTT;

129 ACE2-RT-R: ACCACCCCAACTATCTCTCG;

130 nCoVN-RT-F: CATTGGCATGGAAGTCACAC;

131 nCoVN-RT-R: TCTGCGGTAAGGCTTGAGTT;

132 GAPDH-RT-F: TGGGTGTGAACCATGAGAAG;

133 GAPDH-RT-R: GTGTCGCTGTTGAAGTCAGA.

\section{$134 \quad 2.4$ The proliferation assay}

135 iPSC, iPSC-GFP and iPSC-nCoVN were seeded in 96-well plates with the same cell number. After 13624 hours, CCK-8 reagent was added in the medium to monitor the proliferation rate (Beyotime, 137 C0038). The absorbance at $450 \mathrm{~nm}$ was measured at 24 hours, 42 hours, 48 hours, 60 hours and 72 138 hours by using Varioskan Flash Multimode Reader (Thermo Scientific). The cell-free medium with 139 CCK-8 reagent were used as blank control sets. The data were analyzed and plotted using GraphPad 140 Prism 6.

\section{$141 \quad 2.5$ Immunofluorescence Staining}

142 Cells were fixed with 4\% paraformaldehyde at room temperature for 20 minutes and washed three 143 times with $1 \times$ PBS. Cells were then permeabilized with PBS containing $0.25 \%$ Triton X-100 at room temperature for 10 minutes. After incubating in the blocking buffer (1× PBS with $10 \%$ goat serum), cells were stained with different primary antibodies at $4{ }^{\circ} \mathrm{C}$ overnight. These primary antibodies were [target, dilution, species, company, product number]: Troponin T Cardiac Isoform, 1:100, mouse, Thermo Fisher, MA5-12960; alpha-smooth muscle actin, 1:100, mouse, Bioss, bsm-33187M; S100A4, 1:100, rabbit, Bioss, bs-3759R; SSEA4, 1:250, mouse, Invitrogen, 14-8843-80; TRA-1-81, 1:250, mouse, Invitrogen, 14-8883-80; 6× His Tag, 1:250, mouse, Sangon Biotech, D191001; OCT4, 1:200, mouse, Abcam, ab184665; ACE2, 1:200, rabbit, Bioss, bs-1004R; vimentin, 1:250, rabbit, Bioss, bs-0756R. Cells were washed three times with PBS containing 0.1\% Triton X-100, then incubated with the Alexa Fluor 488 goat anti-mouse or Alexa Fluor 555 goat anti-rabbit IgG secondary antibodies at $37^{\circ} \mathrm{C}$ for 1 hour. Nuclei were labeled with DAPI (4',6-diamidino-2phenylindole, $1 \mu \mathrm{g} / \mathrm{ml}$ ) for 5 minutes. Images were obtained by using the DMi6000 B inverted 
microscope (Leica) or the FV1000 confocal laser scanning microscope (Olympus), then were

156 analyzed by using ImageJ software.

\section{$157 \quad 2.6$ RNA-seq analysis method}

158 RNA-seq was performed by Novogene Co., Ltd. We obtained 151 bp paired-end RNA-seq reads from an Illumina Novaseq instrument, average 23 million read pairs for 3 iPSC-GFP and 3 iPSCnCoVN samples. Adapters and low-quality bases in reads were trimmed by trim_galore (v0.6.5; http://www.bioinformatics.-babraham.ac.uk/projects/trim_galore/). We employed Kallisto (v0.46.0) (Bray et al., 2016) to determine the read count for each transcript and quantified transcript abundance as transcripts per kilobase per million reads mapped (TPM), using gene annotation in the GENCODE database (v32, GRCh38) (Frankish et al., 2019). Then we summed the read counts and TPM of all alternative splicing transcripts of a gene to obtain gene expression levels. We restricted our analysis to 22,201 expressed genes with an average TPM >=1 in either iPSC-GFP or iPSC-nCoVN samples. DESeq2 (v1.26.0) (Love et al., 2014) was used to identify differentially expressed genes (DEGs) (false discovery rate (FDR) $<0.05$ and abs(log2FoldChange) $>3$ ). The pathway analysis was performed by ToppGene Suite (Chen et al., 2009). The raw RNA-seq data have been deposited in $\mathrm{GEO}$, and the accession number is pending.

We took gene expression values (i.e. $\log _{2}(\mathrm{TPM})$ ) in iPSC/ESC and fibroblast from ENCODE (Consortium, 2012; Davis et al., 2018). Combining with our RNA-seq data, quantile normalization (Bolstad et al., 2003) was performed, and then ComBat (Johnson et al., 2007) was used to remove the batch correction. We selected the top 1000 genes with the largest variance to calculate the correlation coefficients between samples. The heatmap was generated by pheatmap function in $\mathrm{R}$.

\subsection{Statistic}

177 Values were expressed as mean \pm SD (standard deviation). Statistical significances were evaluated using one-way ANOVA with Bonferroni correction or Student's T-Test. $P<0.05$ was considered statistically significant.

\section{Results}

\subsection{ACE2 was expressed in various of stem cells}

As ACE2 was the major receptor of SARS-CoV-2 on the cell membrane (Hoffmann et al., 2020; Wrapp et al., 2020), we first examined whether ACE2 was expressed in the stem cells. Thanks to the gene expression data collection in Gene Expression Omnibus (GEO, https://www.ncbi.nlm.nih.gov/geo/), it was convenient to analyze the ACE2 expression profiles in sorts of stem cells. Figure 1A showed the ACE2 expression values in different stem cells from different projects, including human embryonic stem cells (Kim et al., 2014), iPSC (Yang et al., 2014), human epithelial stem cells (Yang et al., 2014), human adipose stem cells (Onate et al., 2013), human hematopoietic stem cells (Pang et al., 2011), and human mesenchymal stem cells (Bernstein et al., 2010). The expression values of a housekeeping gene GAPDH were simultaneously collected as controls. ACE2 was expressed in each kind of stem cells, though the expression values were relatively low compared with GAPDH. The reverse transcription-PCR (RT-PCR) results showed that $A C E 2$ was expressed in iPSC, iPSC-derived cardiomyocytes (iPSC-CM) and human coronary artery endothelial cells (HCAEC) (Figure 1B). The images from immunofluorescence assays clearly showed that ACE2 protein was located on the cell membrane of iPSC (Figure 1C), suggesting that 


\subsection{Expression of nCoVN changed the morphology of iPSC}

198 To study whether physiological activities in iPSC were disturbed by nCoVN, a human induced pluripotent stem cell line (iPSC-nCoVN) in which the expression of nCoVN could be modulated by a Tet-On system was generated by a lentiviral expression system. In this system, nCoVN cDNA sequence (with a $6 \times$ His Tag coding sequence) was conjugated to puromycin resistance gene through a T2A peptide encoding sequence, and the transcription was relied on the induction of tetracycline or doxycycline (Dox). After puromycin selection, two single cell clones were seeded in separated wells by manual colony-picking. Sequentially, iPSC-nCoVN were divided into two groups: one was induced by Dox for nCoVN expression (Dox), the other was added with DMSO as a control set (DMSO), meanwhile, a GFP-expressed iPS cell line (iPSC-GFP), in which the expression of GFP was modulated by the same Tet-On system, was used as another control set in the following assays (Figure 2A).

The expression of nCoVN was confirmed at the mRNA and protein levels. The transcriptional level of nCoVN was measured by Real-time PCR in iPSC, Dox, and DMSO groups, and nCoVN expression increased about 267-fold in the Dox group compared with the DMSO group (Figure 2B). The nCoVN protein was detected by using an anti-6x His Tag antibody in cells from the Dox group (Supplementary Figure 1). The proliferation rate was compared among iPSC, iPSC-GFP and Dox groups by using a cell counting kit. The absorbance at $450 \mathrm{~nm}$ (A450) was measured at 24 hours, 42 hours, 48 hours, 60 hours and 72 hours after cell seeding. After three days of cell seeding, Dox group showed a decreased proliferation rate than both of iPSC and iPSC-GFP groups, indicating that nCoVN might hamper the growth and division of iPSC (Figure 2C). This observation was consistent with the previous finding about the nucleocapsid protein of SARS-CoV (Zhou et al., 2008).

We continued to induce nCoVN expression in iPSC. The phase-contrast images of iPSC-nCoVN with a 7-day, a 9-day, and an 11-day inductions (Dox) and counterpart controls (DMSO) were shown in Figure 2D. In the DMSO group, a typical morphology of stem cells with high nucleus/cytoplasm ratio and close cell membrane contacts was observed, while iPSC-nCoVN after a 7-day induction started to exhibit endothelial cell morphological features and lower nucleus/cytoplasm ratio. After a 14-day induction, most of the cells exhibited distinct shapes from wild-type iPSC, such as neuronlike cells, endothelial-like cells and fibroblast-like cells (Figure 2E). These data showed that continuous expression of nCoVN caused obvious morphological changes in iPSC.

\subsection{Expression of nCoVN disabled the pluripotent properties of iPSC}

Next, we examined the pluripotency markers in iPSC and iPSC-nCoVN. The pluripotency markers SSEA4 and TRA-1-81, which were expressed in human embryonic stem cells and iPSC, were widely

230

231

232

233

234

235

236

237

238

239

240 applied in identification of pluripotent stem cells (Abujarour et al., 2013; Trusler et al., 2018). The immunofluorescence staining images illustrated that iPSC-nCoVN completely lost the expression of SSEA4 and TRA-1-81, namely, iPSC-nCoVN lost the pluripotency in the presence of nCoVN (Supplementary Figure 2A, B). We traced the expression of TRA-1-81 in iPSC-nCoVN with a 2-day, a 4-day, a 6-day, and an 8-day inductions (Figure 3A). On Day 2, TRA-1-81 was still expressed in iPSC-nCoVN; however, from Day 4, TRA-1-81 was not detectable in most of the cells, suggesting that the pluripotent fate of iPSC-nCoVN was determined in the first 4 days. To further test the pluripotency in iPSC and iPSC-nCoVN, we directly differentiated these cells to cardiomyocytes by using a routine protocol, and the differentiation assays were performed under the same conditions. As expected, the differentiation efficiency could reach $60 \%$ in iPSC; however, on differentiation day 12 , only a very small portion of cells from iPSC-nCoVN were expressed cardiac Troponin $\mathrm{T}$, 
accompanied by many cell deaths (Figure 3B, C). This differentiation assay provided solid evidence that the pluripotency maintenance of iPSC-nCoVN was disrupted by nCoVN.

\section{$243 \quad 3.4 \quad$ Long-term expression of nCoVN drove iPSC to fibroblast}

244 Since the pluripotency lost due to short-term expression of nCoVN, we are extremely interested in the cell fate of iPSC-nCoVN under long-term expression of nCoVN. After a 28-day induction in the stem cell culture medium, some spindle-shaped iPSC-nCoVN, which exhibited a typical fibroblast morphological feature, were observed (Supplementary Figure 2C). The antibodies against fibroblast markers vimentin, alpha-smooth muscle actin ( $\alpha$-SMA) and S100A4 were used to verify the cell type of these fibroblast-like cells. The results from immunofluorescence assays confirmed that these markers were expressed in nCoVN-expressing cells (Figure 4A, B, C; Supplementary Figure 2D). To further investigate the transcriptomic profiles of iPSC-nCoVN under the long-term nCoVN expression, doxycycline-induced iPSC-nCoVN and iPSC-GFP for 30 days were applied to RNA-seq. Through differentially express analysis, iPSC-nCoVN showed a dramatic gene expression change comparing with iPSC-GFP (Supplementary Table). Totally, 3,080 genes were significantly differentially expressed (FDR $<0.05, \mid \log 2$ FoldChange $\mid>3$ ). Among them, the down-regulated genes in iPSC-nCoVN were most significantly enriched with proliferation and stem cell related pathways, including the Yamanaka factors-associated genes, such as POU5F1, LIN28A, NANOG, and SOX2 (with a 790-fold, a 2306-fold, a 253-fold, and an 18-fold decrease, respectively) (Figure 4E); while the extracellular matrix and extracellular matrix-associated pathway was the most significantly enriched pathway in the up-regulated genes (Figure 4F). Next, we used RNA-seq data from the ENCODE project to evaluate the cell type of iPSC-nCoVN. Comparing with the transcriptome of the pluripotent stem cells and fibroblast in the ENCODE project (Consortium, 2012; Davis et al., 2018), iPSC-GFP samples were clustered with H7 and GM23338, which were the embryonic stem cells (ESC) and iPSC, respectively; while iPSC-nCoVN samples were clustered with multiple kinds of fibroblast (Figure 4G). Furthermore, iPSC-nCoVN with a 40-day induction, which were kept culturing in the stem cell medium, were totally differentiated to fibroblast (Figure 4D).

\section{Discussion}

According to the current knowledge about the life cycle of SARS-CoV, the nucleocapsid protein was

269

270

271 translated by the host cell translation protein synthesis machinery (McBride et al., 2014; Song et al., 2019), and was localized mainly in the cytoplasm (Rowland et al., 2005). The primary function of nucleocapsid protein was to package the viral genome into nucleocapsids to protect the genomic RNA (McBride et al., 2014). During the formation of nucleocapsids, numerous nucleocapsid proteins bound to the viral RNA and started oligomerization. The viral reproductive strategies would synthesize nucleocapsid proteins as many as possible to meet the requirements of viral assembly, which meant the nucleocapsid proteins were overproduced. The findings that redundant nucleocapsid proteins interfered with the normal physiology of host cells were reported (Surjit et al., 2004; Zhao et al., 2006; Zhang et al., 2007a; Zhao et al., 2008; Zhou et al., 2008; Hu et al., 2017). In this study, we first presented that nCoVN abolished pluripotency and reduced the proliferation rate in human induced pluripotent stem cells. Long-term expression of $\mathrm{nCoVN}$ drove iPSC to fibroblast in spite of using the stem cell culture conditions. It was reported that the nucleocapsid protein of SARS-CoV facilitated TGF- $\beta$-induced PAI-1 expression to promote lung fibrosis (Zhao et al., 2008), which was also the possible pathway that nCoVN turned iPSC to fibroblast.

284

The time-course assays showed that the pluripotency marker disappeared in four days after nCoVN expression. This finding might be applied to a cell-based chemical screening model, in which the 
candidate chemicals with potential ability to halt the iPSC differentiation caused by nCoVN are easily identified. More importantly, SARS-CoV-2 is not necessary in this model, which means it could be used in the routine laboratories and applied to high-throughput equipment with less risk.

In addition, how nCoVN breaks the pluripotency maintenance of iPSC is still a riddle. The pluripotency maintenance in stem cells requires delicate regulations to maintain the balance of pluripotency gene expression in a complicated network. Since nCoVN can bind RNAs, it is possible that nCoVN suppresses the key pluripotency gene's translation through occupying the particular sites of RNAs. Although the mechanism is unknown, the toxic effects of $\mathrm{nCoVN}$ are clear, which reminds us that SARS-CoV-2 might impair the reproductive system and hematopoietic system. In conclusion, we first reported expressing nCoVN could totally change the cell fate of iPSC, which provided new clues to help people fighting against the virus.

\section{$5 \quad$ Conflict of Interest}

Author Zebin Lin, Jinlian Mai, Lishi Zhou, and Bin Lin were employed by the company Guangdong Beating Origin Regenerative Medicine Co. Ltd. The remaining authors declare that the research was conducted in the absence of any commercial or financial relationships that could be construed as a potential conflict of interest.

\section{Author Contributions}

ZL, ZW, PW, and BL had substantial contributions to the design of the paper; ZL, ZW, JM, LZ, YQ, and TC performed the experiments and analysed the data; $\mathrm{ZC}$ provided critical suggestions to improve the paper; ZL, ZW, PW, and BL wrote the manuscript. All authors (ZL, ZW, JM, LZ, YQ, TC, ZC, PW, and BL) had read and approved the final manuscript.

\section{$7 \quad$ Funding}

This work was supported by grants from the National Natural Science Foundation of China (NSFC) to Ping Wang (31900812).

\section{Acknowledgments}

This work is dedicated to all the medical staff who are still fighting against COVID-19 in China. Your efforts make us safer.

\section{$9 \quad$ Figure legends}

Figure 1. ACE2 was expressed in human stem cells. (A) Expression values of ACE2 and GAPDH derived from the Gene Expression Omnibus database. (B) Images from agarose gel electrophoresis for analyzing the Reverse transcription-PCR products. ACE2 was expressed in iPSC, iPSC-CM and HCAEC. iPSC, human induced pluripotent stem cell; iPSC-CM, human induced pluripotent stem cell-derived cardiomyocyte; HCAEC, human coronary artery endothelial cell; NC, negative control. (C) Representative immunofluorescent staining images of ACE2 (red) and the pluripotency marker OCT4 (green) in iPSC. The cell nuclei were stained by DAPI (blue). The scale bar represents $10 \mu \mathrm{m}$.

Figure 2. nCoVN affected the proliferation and morphology of iPSC. (A) Schematic diagram illustrating the generation of iPSC-nCoVN and controls. Purple cells indicated iPSC without nCoVN expression, while yellow cells indicated iPSC with nCoVN expression. Green cells were iPSC stably 
323

324

325

326

327

328

329

330

331

332

333

334

335

336

337

338

339

340

341

342

343

344

345

346

347

348

349

350

351

352

353

354

355

356

357

358

359

360

361

362

363

364 expressing GFP under the doxycycline induction. (B) The mRNA expression level of nCoVN was significantly elevated in iPSC-nCoVN for a long-term induction $(\mathrm{n}=3)$. **, $p<0.001$. (C) The time course of cellular proliferation from iPSC, iPSC-GFP and iPSC-nCoVN $(n=6)$. At 72 hours after cell seeding, the values of A450 were significantly increased in iPSC and iPSC-GFP groups. **, $p<0.001$. (D) Representative phase-contrast images from nCoVN-positive cells (Dox group) and control cells (DMSO group) after a 7-day, a 9-day, and an 11-day inductions. The scale bar is $50 \mu \mathrm{m}$. (E) Representative phase-contrast images of iPSC-nCoVN after a 14-day induction show detailed morphological alterations. Images were taken under objectives with $10 \times, 20 \times$, and $40 \times$ magnifications (from left to right panels). White dashed line boxes indicate the regions that are magnified in the right panel. The scale bar represents $50 \mu \mathrm{m}$.

Figure 3. iPSC-nCoVN lost the pluripotency. (A) Representative immunofluorescent staining images of pluripotency marker TRA-1-81 (green) in iPSC-nCoVN for a 2-day, a 4-day, a 6-day, and an 8day inductions. The cell nuclei were stained by DAPI (blue). The scale bar represents $50 \mu \mathrm{m}$. (B) Representative immunofluorescent staining images of cardiomyocyte marker cardiac Troponin $\mathrm{T}$ (Red) in iPSC- and iPSC-nCoVN-derived cardiomyocytes. The cell nuclei were stained by DAPI (blue). The scale bar represents $50 \mu \mathrm{m}$. (C) The cardiac differentiation efficiency of iPSC and iPSCnCoVN. Images taken from (B) were analyzed by using ImageJ software. The efficiency was calculated as the portion of cardiac Troponin T positive cells in all the cells. Approximately 6,000 cells were counted in each group. ${ }^{* *}, p<0.001$.

Figure 4. Long-term expression of $\mathrm{nCoVN}$ turned iPSC to fibroblast. (A-C) Representative immunofluorescent staining images of vimentin (green), S100A4 (green), and $\alpha$-SMA (green) in iPSC-nCoVN after a 10-day induction. The cell nuclei were stained by DAPI (blue). The scale bars represent $10 \mu \mathrm{m}$. (D) The representative bright field image of cells after a 40-day nCoVN expression. The morphology exhibits typical fibroblast features. The scale bar represents $50 \mu \mathrm{m}$. (E) The two most significantly enriched pathways in the down-regulated genes. The histogram shows the significance of the two pathways by using $-\log _{10}$ (q values with Bonferroni correction). The Log 2fold change values of a total of 14 genes in these two pathways were exhibited by the heatmap. (F) The three most significantly enriched pathways in the up-regulated genes. The histogram shows the significance of the three pathways by using $-\log _{10}$ (q values with Bonferroni correction). The Log 2fold change values of the top 20 up-regulated genes in these pathways were exhibited by the heatmap. (G) The heatmap of the correlation coefficients among iPSC-GFP, iPSC-nCoVN, ESC/iPSC and fibroblast from the ENCODE project. The 1000 most variable genes in the samples were used to calculate the correlation coefficients. iPSC-GFP and iPSC-nCoVN clustered with pluripotent stem cell and fibroblast, respectively.

\section{References}

Abujarour, R., Valamehr, B., Robinson, M., Rezner, B., Vranceanu, F., and Flynn, P. (2013). Optimized surface markers for the prospective isolation of high-quality hiPSCs using flow cytometry selection. Sci Rep 3, 1179. doi: 10.1038/srep01179.

Bernstein, P., Sticht, C., Jacobi, A., Liebers, C., Manthey, S., and Stiehler, M. (2010). Expression pattern differences between osteoarthritic chondrocytes and mesenchymal stem cells during chondrogenic differentiation. Osteoarthritis Cartilage 18(12), 1596-1607. doi: 10.1016/j.joca.2010.09.007. 
Bolstad, B.M., Irizarry, R.A., Astrand, M., and Speed, T.P. (2003). A comparison of normalization methods for high density oligonucleotide array data based on variance and bias. Bioinformatics 19(2), 185-193. doi: 10.1093/bioinformatics/19.2.185.

Bray, N.L., Pimentel, H., Melsted, P., and Pachter, L. (2016). Near-optimal probabilistic RNA-seq quantification. Nat Biotechnol 34(5), 525-527. doi: 10.1038/nbt.3519.

Chang, C.K., Hou, M.H., Chang, C.F., Hsiao, C.D., and Huang, T.H. (2014). The SARS coronavirus nucleocapsid protein--forms and functions. Antiviral Res 103, 39-50. doi: 10.1016/j.antiviral.2013.12.009.

Chang, C.K., Lo, S.C., Wang, Y.S., and Hou, M.H. (2016). Recent insights into the development of therapeutics against coronavirus diseases by targeting N protein. Drug Discov Today 21(4), 562-572. doi: 10.1016/j.drudis.2015.11.015.

Chen, J., Bardes, E.E., Aronow, B.J., and Jegga, A.G. (2009). ToppGene Suite for gene list enrichment analysis and candidate gene prioritization. Nucleic Acids Res 37(Web Server issue), W305-311. doi: 10.1093/nar/gkp427.

Cheung, Y.K., Cheng, S.C., Sin, F.W., Chan, K.T., and Xie, Y. (2008). Investigation of immunogenic T-cell epitopes in SARS virus nucleocapsid protein and their role in the prevention and treatment of SARS infection. Hong Kong Med J 14 Suppl 4, 27-30.

Chinese, S.M.E.C. (2004). Molecular evolution of the SARS coronavirus during the course of the SARS epidemic in China. Science 303(5664), 1666-1669. doi: 10.1126/science.1092002.

Consortium, E.P. (2012). An integrated encyclopedia of DNA elements in the human genome. Nature 489(7414), 57-74. doi: 10.1038/nature11247.

Das, D., Kammila, S., and Suresh, M.R. (2010). Development, characterization, and application of monoclonal antibodies against severe acute respiratory syndrome coronavirus nucleocapsid protein. Clin Vaccine Immunol 17(12), 2033-2036. doi: 10.1128/CVI.00293-10.

Davis, C.A., Hitz, B.C., Sloan, C.A., Chan, E.T., Davidson, J.M., Gabdank, I., et al. (2018). The Encyclopedia of DNA elements (ENCODE): data portal update. Nucleic Acids Res 46(D1), D794-D801. doi: 10.1093/nar/gkx1081.

Frankish, A., Diekhans, M., Ferreira, A.M., Johnson, R., Jungreis, I., Loveland, J., et al. (2019). GENCODE reference annotation for the human and mouse genomes. Nucleic Acids Res 47(D1), D766-D773. doi: 10.1093/nar/gky955.

He, R., Leeson, A., Andonov, A., Li, Y., Bastien, N., Cao, J., et al. (2003). Activation of AP-1 signal transduction pathway by SARS coronavirus nucleocapsid protein. Biochem Biophys Res Commun 311(4), 870-876. doi: 10.1016/j.bbrc.2003.10.075.

Hoffmann, M., Kleine-Weber, H., Schroeder, S., Kruger, N., Herrler, T., Erichsen, S., et al. (2020). SARS-CoV-2 Cell Entry Depends on ACE2 and TMPRSS2 and Is Blocked by a Clinically Proven Protease Inhibitor. Cell. doi: 10.1016/j.cell.2020.02.052.

Hu, Y., Li, W., Gao, T., Cui, Y., Jin, Y., Li, P., et al. (2017). The Severe Acute Respiratory Syndrome Coronavirus Nucleocapsid Inhibits Type I Interferon Production by Interfering with TRIM25-Mediated RIG-I Ubiquitination. J Virol 91(8). doi: 10.1128/JVI.02143-16.

Jiang, W., Hua, R., Wei, M., Li, C., Qiu, Z., Yang, X., et al. (2015). An optimized method for hightiter lentivirus preparations without ultracentrifugation. Sci Rep 5, 13875. doi: 10.1038/srep13875. 
407

408

409

410

411

412

413

414

415

416

417

418

419

420

421

422

423

424

425

426

427

428

429

430

431

432

433

434

435

436

437

438

439

440

441

442

443

444

445

446

447

Johnson, W.E., Li, C., and Rabinovic, A. (2007). Adjusting batch effects in microarray expression data using empirical Bayes methods. Biostatistics 8(1), 118-127. doi: 10.1093/biostatistics/kxj037.

Kim, J.J., Khalid, O., Namazi, A., Tu, T.G., Elie, O., Lee, C., et al. (2014). Discovery of consensus gene signature and intermodular connectivity defining self-renewal of human embryonic stem cells. Stem Cells 32(6), 1468-1479. doi: 10.1002/stem.1675.

Lin, B., Lin, X., Stachel, M., Wang, E., Luo, Y., Lader, J., et al. (2017). Culture in Glucose-Depleted Medium Supplemented with Fatty Acid and 3,3',5-Triiodo-1-Thyronine Facilitates Purification and Maturation of Human Pluripotent Stem Cell-Derived Cardiomyocytes. Front Endocrinol (Lausanne) 8, 253. doi: 10.3389/fendo.2017.00253.

Love, M.I., Huber, W., and Anders, S. (2014). Moderated estimation of fold change and dispersion for RNA-seq data with DESeq2. Genome Biol 15(12), 550. doi: 10.1186/s13059-014-0550-8.

Lu, R., Zhao, X., Li, J., Niu, P., Yang, B., Wu, H., et al. (2020). Genomic characterisation and epidemiology of 2019 novel coronavirus: implications for virus origins and receptor binding. Lancet 395(10224), 565-574. doi: 10.1016/S0140-6736(20)30251-8.

McBride, R., van Zyl, M., and Fielding, B.C. (2014). The coronavirus nucleocapsid is a multifunctional protein. Viruses 6(8), 2991-3018. doi: 10.3390/v6082991.

Onate, B., Vilahur, G., Camino-Lopez, S., Diez-Caballero, A., Ballesta-Lopez, C., Ybarra, J., et al. (2013). Stem cells isolated from adipose tissue of obese patients show changes in their transcriptomic profile that indicate loss in stemcellness and increased commitment to an adipocyte-like phenotype. BMC Genomics 14, 625. doi: 10.1186/1471-2164-14-625.

Pang, W.W., Price, E.A., Sahoo, D., Beerman, I., Maloney, W.J., Rossi, D.J., et al. (2011). Human bone marrow hematopoietic stem cells are increased in frequency and myeloid-biased with age. Proc Natl Acad Sci U S A 108(50), 20012-20017. doi: 10.1073/pnas.1116110108.

Rowland, R.R., Chauhan, V., Fang, Y., Pekosz, A., Kerrigan, M., and Burton, M.D. (2005). Intracellular localization of the severe acute respiratory syndrome coronavirus nucleocapsid protein: absence of nucleolar accumulation during infection and after expression as a recombinant protein in vero cells. J Virol 79(17), 11507-11512. doi: 10.1128/JVI.79.17.11507-11512.2005.

Severance, E.G., Bossis, I., Dickerson, F.B., Stallings, C.R., Origoni, A.E., Sullens, A., et al. (2008). Development of a nucleocapsid-based human coronavirus immunoassay and estimates of individuals exposed to coronavirus in a U.S. metropolitan population. Clin Vaccine Immunol 15(12), 1805-1810. doi: 10.1128/CVI.00124-08.

Shalem, O., Sanjana, N.E., Hartenian, E., Shi, X., Scott, D.A., Mikkelson, T., et al. (2014). Genomescale CRISPR-Cas9 knockout screening in human cells. Science 343(6166), 84-87. doi: 10.1126/science.1247005.

Shekhar, A., Lin, X., Lin, B., Liu, F.Y., Zhang, J., Khodadadi-Jamayran, A., et al. (2018). ETV1 activates a rapid conduction transcriptional program in rodent and human cardiomyocytes. Sci Rep 8(1), 9944. doi: 10.1038/s41598-018-28239-7.

Song, Z., Xu, Y., Bao, L., Zhang, L., Yu, P., Qu, Y., et al. (2019). From SARS to MERS, Thrusting Coronaviruses into the Spotlight. Viruses 11(1). doi: 10.3390/v11010059. 
Suresh, M.R., Bhatnagar, P.K., and Das, D. (2008). Molecular targets for diagnostics and therapeutics of severe acute respiratory syndrome (SARS-CoV). J Pharm Pharm Sci 11(2), 1s-13s. doi: 10.18433/j3j019.

Surjit, M., Liu, B., Jameel, S., Chow, V.T., and Lal, S.K. (2004). The SARS coronavirus nucleocapsid protein induces actin reorganization and apoptosis in COS-1 cells in the absence of growth factors. Biochem J 383(Pt 1), 13-18. doi: 10.1042/BJ20040984.

Trusler, O., Huang, Z., Goodwin, J., and Laslett, A.L. (2018). Cell surface markers for the identification and study of human naive pluripotent stem cells. Stem Cell Res 26, 36-43. doi: 10.1016/j.scr.2017.11.017.

Wrapp, D., Wang, N., Corbett, K.S., Goldsmith, J.A., Hsieh, C.L., Abiona, O., et al. (2020). CryoEM structure of the 2019-nCoV spike in the prefusion conformation. Science 367(6483), 1260-1263. doi: 10.1126/science.abb2507.

Wu, A., Peng, Y., Huang, B., Ding, X., Wang, X., Niu, P., et al. (2020). Genome Composition and Divergence of the Novel Coronavirus (2019-nCoV) Originating in China. Cell Host Microbe 27(3), 325-328. doi: 10.1016/j.chom.2020.02.001.

Xu, X., P., C., J., W., J., F., H., Z., X., L., et al. (2020). Evolution of the novel coronavirus from the ongoing Wuhan outbreak and modeling of its spike protein for risk of human transmission. Sci China Life Sci 63. doi: https://doi.org/10.1007/s11427-020-1637-5.

Yang, R., Zheng, Y., Burrows, M., Liu, S., Wei, Z., Nace, A., et al. (2014). Generation of folliculogenic human epithelial stem cells from induced pluripotent stem cells. Nat Commun 5, 3071. doi: 10.1038/ncomms4071.

Zhang, L., Wei, L., Jiang, D., Wang, J., Cong, X., and Fei, R. (2007a). SARS-CoV nucleocapsid protein induced apoptosis of COS-1 mediated by the mitochondrial pathway. Artif Cells Blood Substit Immobil Biotechnol 35(2), 237-253. doi: 10.1080/10731190601188422.

Zhang, X., Wu, K., Wang, D., Yue, X., Song, D., Zhu, Y., et al. (2007b). Nucleocapsid protein of SARS-CoV activates interleukin-6 expression through cellular transcription factor NFkappaB. Virology 365(2), 324-335. doi: 10.1016/j.virol.2007.04.009.

Zhao, G., Shi, S.Q., Yang, Y., and Peng, J.P. (2006). M and N proteins of SARS coronavirus induce apoptosis in HPF cells. Cell Biol Toxicol 22(5), 313-322. doi: 10.1007/s10565-006-0077-1.

Zhao, X., Nicholls, J.M., and Chen, Y.G. (2008). Severe acute respiratory syndrome-associated coronavirus nucleocapsid protein interacts with Smad3 and modulates transforming growth factor-beta signaling. J Biol Chem 283(6), 3272-3280. doi: 10.1074/jbc.M708033200.

Zhou, B., Liu, J., Wang, Q., Liu, X., Li, X., Li, P., et al. (2008). The nucleocapsid protein of severe acute respiratory syndrome coronavirus inhibits cell cytokinesis and proliferation by interacting with translation elongation factor 1alpha. J Virol 82(14), 6962-6971. doi: 10.1128/JVI.00133-08. 


\begin{tabular}{|c|c|c|c|c|}
\hline \multirow{4}{*}{ Dataset } & Sample & Cell Line & ACE2 Ave Value & GAPDH Ave Value \\
\cline { 2 - 5 } GDS5408 & GSM1309417 & human embryonic stem cell H1 1 & 25.61 & 24162.72 \\
\cline { 2 - 5 } & GSM1309418 & human embryonic stem cell H1 2 & 30.16 & 26123.47 \\
\cline { 2 - 5 } & GSM1309421 & human embryonic stem cell H9 1 & 39.93 & 24325.00 \\
\cline { 2 - 5 } & GSM1309422 & human embryonic stem cell H9 2 & 27.90 & 23177.18 \\
\hline \multirow{4}{*}{ GDS5638 } & GSM1235179 & human induced pluripotent stem cell 1 & 62.92 & 29904.83 \\
\cline { 2 - 5 } & GSM1235180 & human induced pluripotent stem cell 2 & 76.38 & 30926.80 \\
\cline { 2 - 5 } & GSM1235184 & human epithelial stem cell 1 & 67.86 & 15493.84 \\
\cline { 2 - 5 } & GSM1235185 & human epithelial stem cell 2 & 70.71 & 23540.97 \\
\hline \multirow{3}{*}{ GDS5056 } & GSM1187676 & human adipose stem cell 1 & 5.77 & 14.29 \\
\cline { 2 - 5 } & GSM1187677 & human adipose stem cell 2 & 5.72 & 14.31 \\
\cline { 2 - 5 } & GSM1187678 & human adipose stem cell 3 & 5.87 & 14.34 \\
\hline \multirow{3}{*}{ GDS3942 } & GSM812988 & human hematopoietic stem cell 1 & 3.77 & 12.47 \\
\cline { 2 - 5 } & GSM812989 & human hematopoietic stem cell 2 & 4.71 & 12.54 \\
\cline { 2 - 5 } & GSM812990 & human hematopoietic stem cell 3 & 4.20 & 12.61 \\
\hline GDS3785 & GSM490983 & human mesenchymal stem cell & 5.47 & 12.29 \\
\hline
\end{tabular}

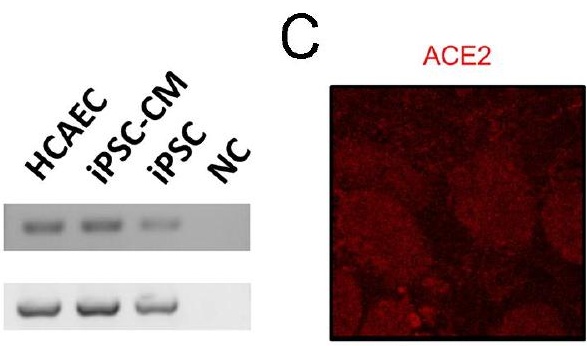

OCT4

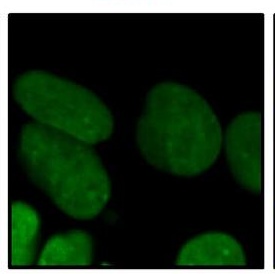

DAPI

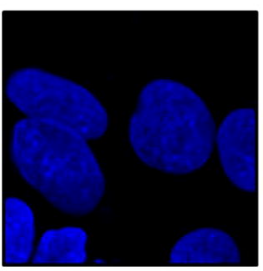

Merge

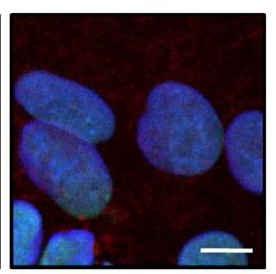







A

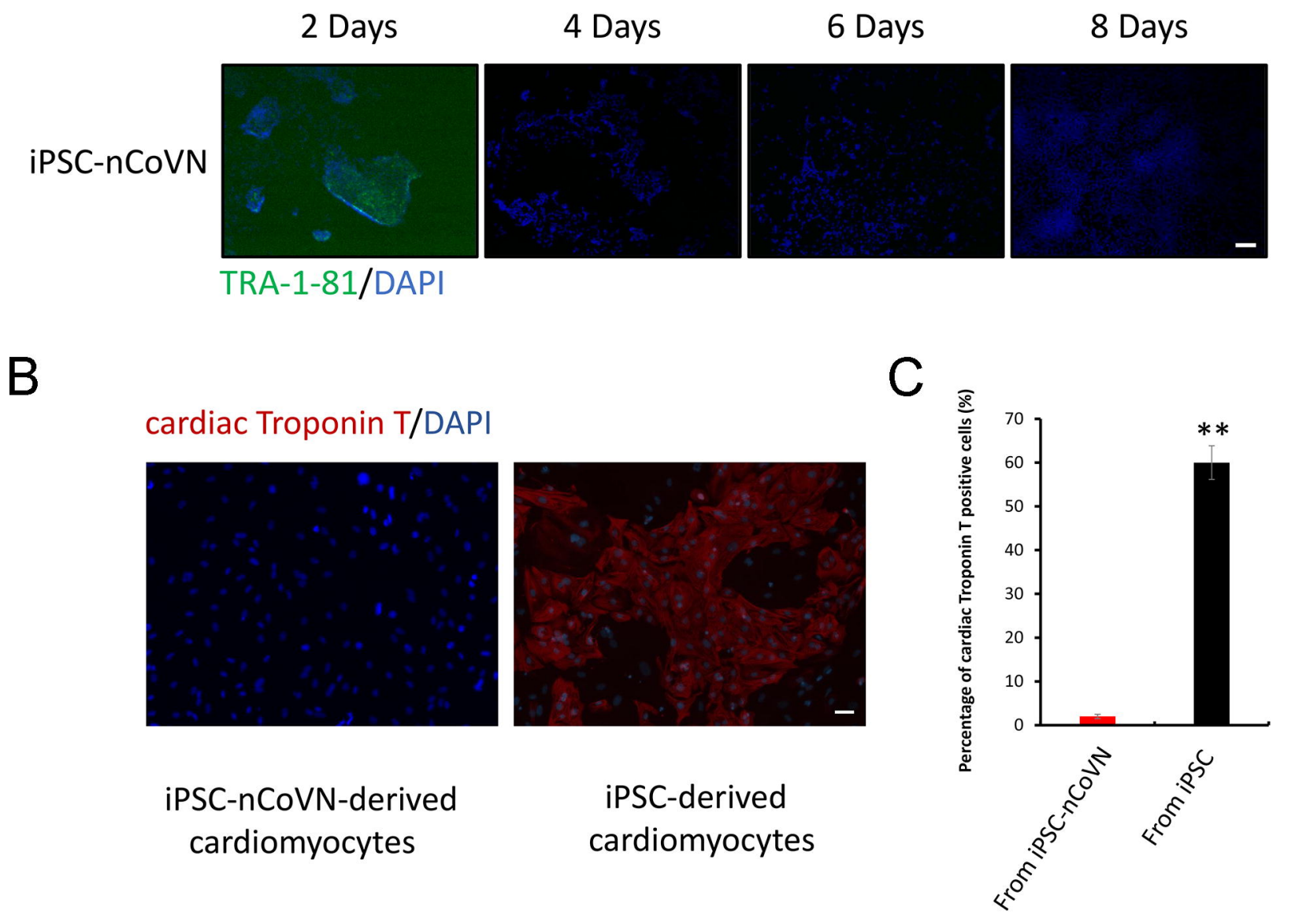




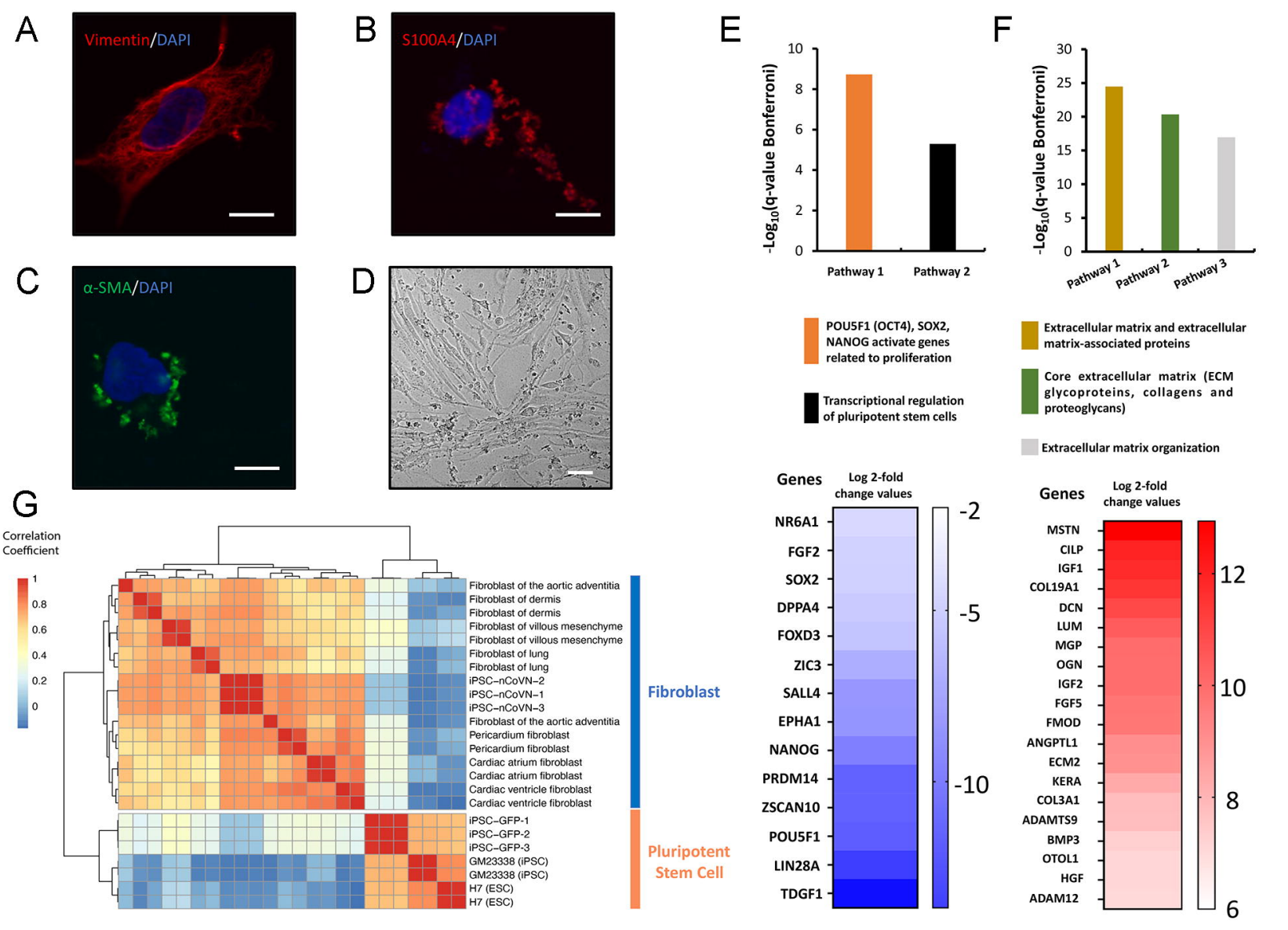

